\title{
Die feline hypertrophe Kardiomyopathie
}

\author{
Markus Killich
}

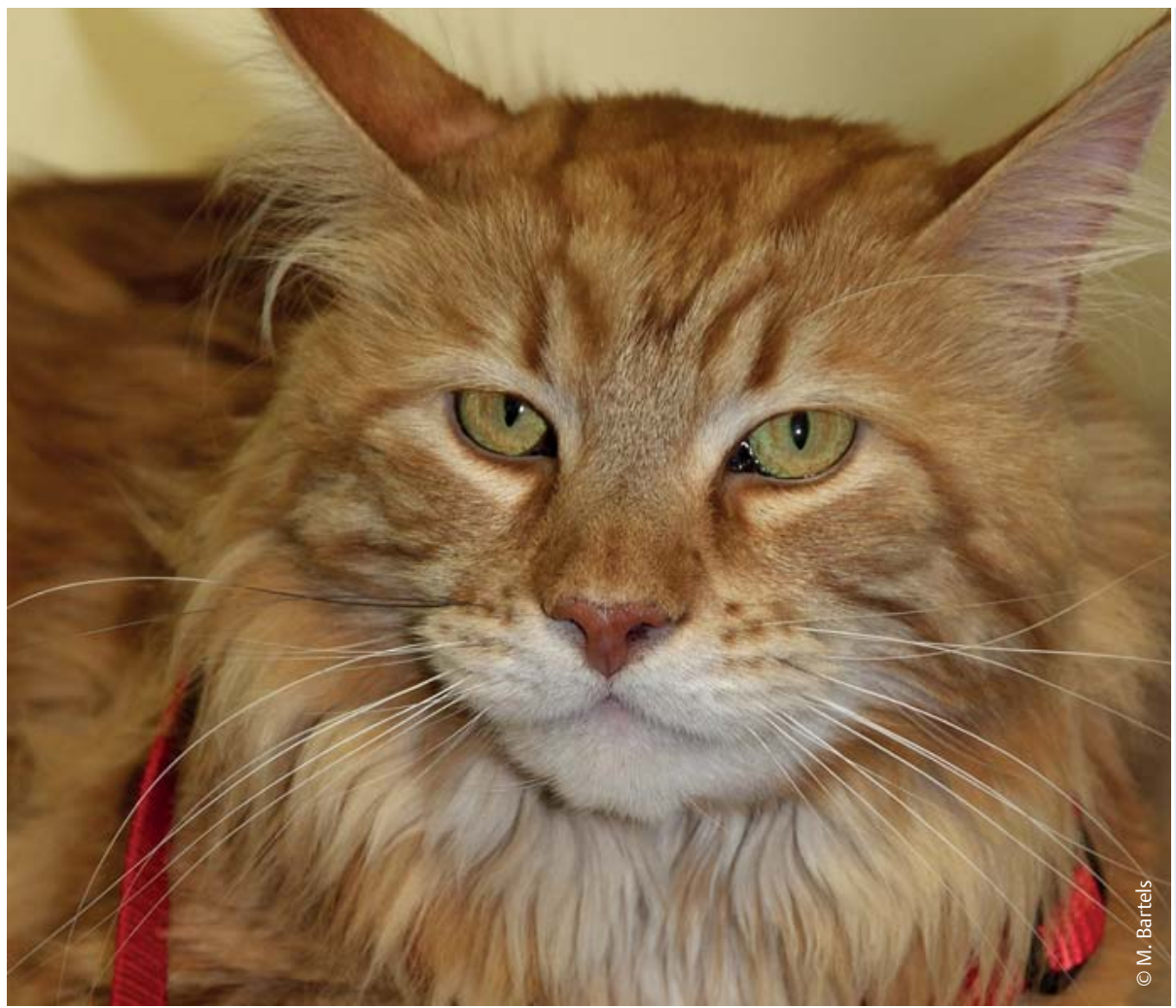

Katzen sind Meister im Verstecken ihrer Symptome. Bei welchen Patienten sollte der behandelnde Tierarzt dennoch frühzeitig an ein Herzproblem denken und welche Untersuchungen sind bei einem Verdacht hilfreich?

Der Begriff „Kardiomyopathie“ beschreibt eine Gruppe von primären Herzmuskelerkrankungen, deren Auslöser auf zellulärer Ebene im Herzmuskel liegt. Die hypertrophe Kardiomyopathie (HKM; engl. hypertrophic cardiomyopathy: HCM) ist dabei die häufigste feline Herzmuskelerkrankung. Gekennzeichnet ist die HCM durch eine Verdickung oder konzentrische Hypertrophie der Kammermuskulatur. Die Erkrankung wird auch beim Menschen beobachtet und ist hier bei unter 35-jährigen Haupt- ursache für plötzliche Todesfälle während sportlicher Aktivitäten. Bei Hunden ist die primäre HCM dagegen äußerst selten.

\section{Ätiologie und Pathogenese}

Die HCM ist eine primäre Kardiomyopathie, deren Hauptmerkmal eine Kammerwandverdickung oder konzentrische Hypertrophie ist. Primär bedeutet, dass die Ursache für die Veränderung im Herzmuskel selbst liegt.

Im Gegensatz dazu steht die sekundäre Kardiomyopathie oder besser sekundäre Hypertrophie. Sekundäre Konditionen, die zu einer Verdickung der Kammermuskulatur führen können, sind

- Aortenstenose

- systemische Hypertension
- Hyperthyreose

- Akromegalie (selten)

Eine Dehydratation kann zu einer sog. Pseudohypertrophie führen. Das echokardiografische Bild gleicht dem einer HCM, nach Rehydrierung des Patienten finden sich jedoch normale Kammerdimensionen.

Ursache für die HCM ist ein Gen-Defekt, der eine strukturelle und funktionelle Dysfunktion kardialer Sarkomere hervorruft. Der Gen-Defekt ist entweder hereditär familiär bedingt oder resultiert aus einer spontanen De-novo-Mutation. Beim Menschen sind etwa $66 \%$ hereditär familiär, entsprechende Zahlen bei der Katze liegen nicht vor, dürften aber ähnlich sein. Beim Menschen sind über 250 verschiedene Gen-Defekte als Ursache für die HCM beschrieben. 


\section{Prävalenz und Signalement}

Kardiomyopathien stellen die häufigsten Herzerkrankungen bei Katzen dar. Von den Kardiomyopathien ist die HCM wiederum die häufigste Form.

$\mathrm{Zu}$ den prädisponierten Katzenrassen zählen:

- Bengalen

- Britisch Kurzhaar

- Europäisch Kurzhaar

- Maine Coon

- Perser

- Norwegische Waldkatzen

Die Prävalenz bei klinisch asymptomatischen Tieren liegt je nach Rasse zwischen 10 und 27\% [6,7,16]. Bei den orientalischen Rassen wie beispielsweise Siam-Katzen ist die HCM dagegen selten.

Die Krankheit tritt in allen Altersgruppen auf - das Alter erkrankter Patienten liegt zwischen 6 Monaten bis 16 Jahren.

Orientalische Katzenrassen erkranken nur sehr selten an HCM.

\section{Historie und klinische Präsentation}

Der klinische Verlauf ist durch eine meist mehrere Jahre andauernde, asymptomatische okkulte Phase gekennzeichnet, die für den Besitzer zumeist gar nicht und für den Tierarzt nur mittels bildgebender Verfahren wie der Echokardiografie oder mittels Biomarkern (z.B. NT-ProBNP) zu erkennen ist [17].

Einige Patienten sterben in dieser Phase am plötzlichen Herztod, gerade Maine Coon oder Europäisch Kurzhaar scheinen hiervon vermehrt betroffen [18]. Ein frühes Warnzeichen für die Erkrankung kann Hecheln sein.

Erste klinische Zeichen treten meist jedoch erst im kongestiven Herzversagen auf. Betroffene Patienten zeigen hier Polypnoe oder Dyspnoe. Eine Besonderheit bei Katzen ist, dass einige herzkranke Patienten zunächst nur durch Anorexie auffallen.

Eine weitere „katzen-spezifische“ Präsentation ist die feline Thrombembolie. Ihre

Gewusst? - Pathophysiologie der HCM

Die HCM ist im Gegensatz zur dilatativen Kardiomyopathie (DCM) primär durch eine diastolische Dysfunktion gekennzeichnet. Die Mutation eines Sarkomer-Proteins, wie beispielsweise des Myocyte Binding Protein C (MYBPC), aktiviert eine Kaskade von intrazellulären Signalwegen an deren Ende eine myozytäre Hypertrophie, eine gesteigerte Kollagen-Synthese sowie eine uneinheitliche Ausrichtung der Herzmuskelzellen (sog. „myocyte disarray“) stehen. Diese Veränderungen erhöhen die Steifigkeit des Ventrikels und resultieren in Verbindung mit einem gestörten intrazellulären Kalzium-Stoffwechsel in einer verminderten Füllfunktion. Durch die verminderte Füllung sinken der kardiale Auswurf und letztlich der systemische Blutdruck.

Direkte Folge ist eine Aktivierung des Renin-Angiotensin-Aldosteron-Systems (RAAS). Durch Resorption von $\mathrm{NaCl}$ und $\mathrm{H}_{2} \mathrm{O}$ an den Nieren wird das zirkulierende Blutvolumen kompensatorisch erhöht, was wiederum zu einer besseren Füllung des Ventrikels führt. Allerdings steigt jetzt der linksventrikuläre Füllungsdruck, was sich in einem linksatrialen Druckanstieg und letztlich einer Vergrößerung des linken Atriums widerspiegelt. Das erkrankte Herz kann das Mehr an Volumen auf Dauer nicht verarbeiten, Folge davon ist im Endstadium ein Rückstau in den Lungenkreislauf. Übersteigt der linksatriale Druck einen Wert von 25 mmHg kommt es zum kongestiven Herzversagen mit Ausbildung eines Lungenödems, Thoraxergusses und/oder Perikardergusses.

Burn-out-Kardiomyopathie. Einige Patienten entwickeln im Verlauf der Erkrankung eine sog. Burn-out-Kardiomyopathie. Hierbei handelt es sich um das Endstadium einer HCM, bei der es u. a. durch ein Auseinandergleiten der Myozyten (engl. myocyte slippage) zu einer Verdünnung der Kammerwände mit einer gleichzeitig hochgradig eingeschränkten systolischen Funktion kommt. Die Burn-out-Kardiomyopathie gleicht im Ultraschall dem Bild einer DCM. Sie ist von einer primären DCM nur dadurch zu unterscheiden, dass bei dem Tier eine HCM als Vorerkrankung bekannt war.

Die HCM ist primär durch eine gestörte diastolische Funktion gekennzeichnet. Im Endstadium einer HCM entwickeln einige wenige Patienten eine Burn-out-Kardiomyopathie, die durch eine hochgradig gestörte systolische Funktion gekennzeichnet ist.

Inzidenz liegt bei Katzen mit HCM bei ca. $18 \%$ [17]. Diese perakut verlaufende Symptomatik trifft den Besitzer zumeist unerwartet, da der Patient bis dato oft symptomfrei und somit für den Besitzer nicht als herzkrank zu erkennen war. Betroffene Katzen können zumeist beide Hintergliedmaßen nicht mehr bewegen und leiden unter hochgradigen Schmerzen. Bei Freiläufern wird zunächst oft ein Autounfall als Ursache vermutet. Die zweithäufigste Lokalisation für eine Thrombembolie ist die rechte Arteria subclavia.

Im Gegensatz zum Hund husten Katzen so gut wie nie kardial bedingt. Bei einer hustenden Katze sollte primär an Lungenparasiten, Asthma oder Lungentumoren gedacht werden.
In der klinischen Untersuchung können auskultatorische Befunde auffallen:

- systolisches Herzgeräusch

- Galopprhythmus

- Herzrhythmusstörungen

Viele Katzen zeigen aber trotz teils häufig schon weit fortgeschrittener Veränderung keine in der klinischen Untersuchung feststellbaren Veränderungen. Auch das Vorliegen eines Herzgeräusches ist nicht beweisend für eine Herzerkrankung, da viele gesunde Katzen durch physiologische Strömungsgeräusche auffallen. Bei Katzen mit Herzgeräusch muss deshalb immer eine echokardiografische Untersuchung durchgeführt werden, um das Vorhandensein einer Herzerkrankung zu bestätigen. 

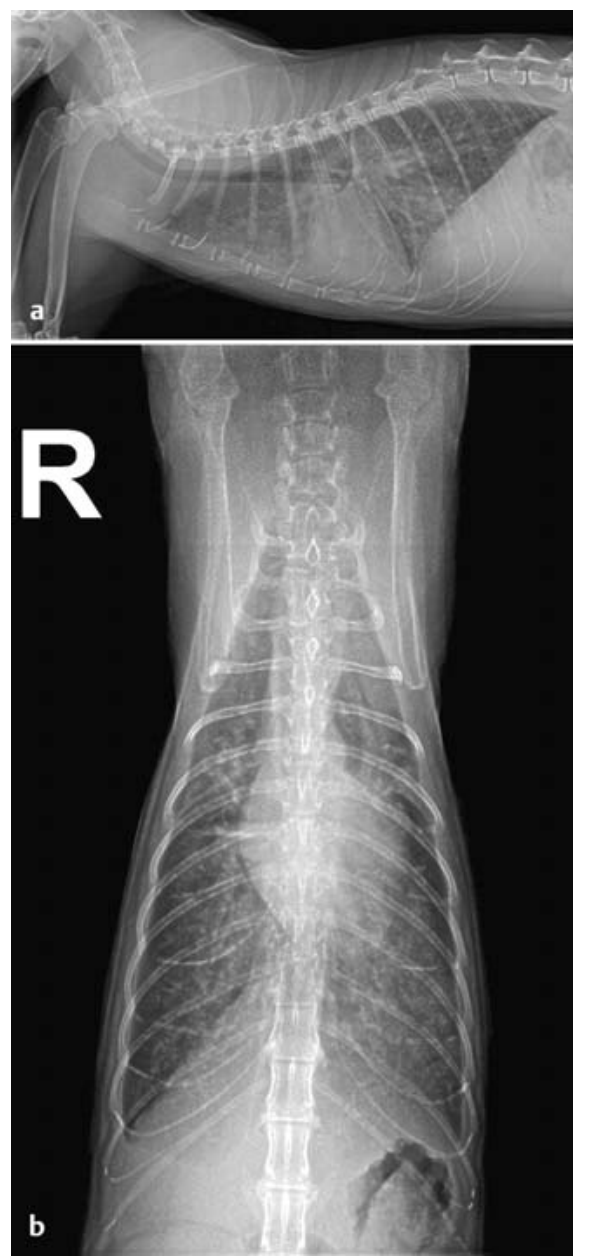

Abb. 1 a und $\mathbf{b}$ Kardiogenes Lungenödem und milder Thoraxerguss in latero-lateraler und ventro-dorsaler Projektion. @ $\mathrm{M}$. Killich

Je jünger ein an HCM erkranktes Tier ist, umso progressiver schreitet die Erkrankung im Allgemeinen fort. Katzen, bei denen die Krankheit dagegen erst in hohem Alter auftritt, zeigen zumeist einen benignen Verlauf [17]. Im kongestiven Herzversagen liegt die mediane überlebenszeit bei etwa 22 Monaten, bei Katzen mit Aortenthrombose dagegen nur bei etwa 6 Monaten [17]. Bei Patienten mit Embolie der Arteria subclavia und erhaltener motorischer Funktion ist die Prognose besser [10].

Männliche Tiere zeigen in der Regel einen progressiveren Verlauf als weibliche. Gerade bei Maine Coon verläuft die Herzerkrankung oft maligne. Kater dieser Rasse kommen häufig bereits mit 2 Jahren ins Endstadium, weibliche Tiere oft schon mit 3 Jahren. Deshalb sollten bei Zuchttieren prädisponierter Rassen auch nach der ersten kardiologischen Screening-Untersuchung jährliche Ultraschallkontrollen erfolgen.

Herzkranke Katzen können, müssen aber keine auskultatorischen Abnormalitäten aufweisen. Das Vorliegen eines Herzgeräusches beweist nicht, dass die Katze herzkrank ist. Katzen aufgrund eines Auskultationsbefundes zu behandeln, ist deshalb nicht korrekt.

\section{Diagnostik}

\section{Elektrokardiografie}

Die Elektrokardiografie ist wenig sensitiv und wenig spezifisch in der Diagnostik der HCM. Die häufigste elektrokardiografische Abnormalität ist der linksanteriore Faszikelblock. Nicht selten werden auch ventrikuläre oder supraventrikuläre Extrasystolen beobachtet. Eine Hypervoltage von mehr als 1,0 mV spricht für eine linksventrikuläre Vergrößerung, ohne jedoch einen Rückschluss auf deren Genese zuzulassen. Äußerst selten ist dagegen das bei Hunden häufig beobachtete Vorhofflimmern. Wird bei einer Katze Vorhofflimmern diagnostiziert, spricht dies für eine massive atriale Dilatation und somit für einen Befund im Endstadium.

Das EKG ist zum Screening einer felinen Kardiomyopathie nicht geeignet.

\section{Röntgen}

Dem Röntgen kommt in der kardialen Diagnostik bei Katzen wesentlich weniger Bedeutung zu als beim Hund. Das in vielen Textbüchern für die ventrodorsale oder dorsoventrale Ebene beschriebene und für die HCM als typisch geltende „valentineshape“ (herzförmige) Herz ist in der Praxis eher selten und nur bei Patienten mit hochgradig dilatiertem Atrium zu erkennen. Katzen mit mildem oder moderatem Befund zeigen in der Regel keine radiologischen Veränderungen. Der obere Wert für die Vertebral Heart Scale (VHS) liegt bei der Katze bei 7,8 Wirbelkörpern.

Zur Diagnosestellung eines kongestiven Herzversagens ist das Röntgen jedoch unerlässlich. Allerdings ist die radiologische Be- 


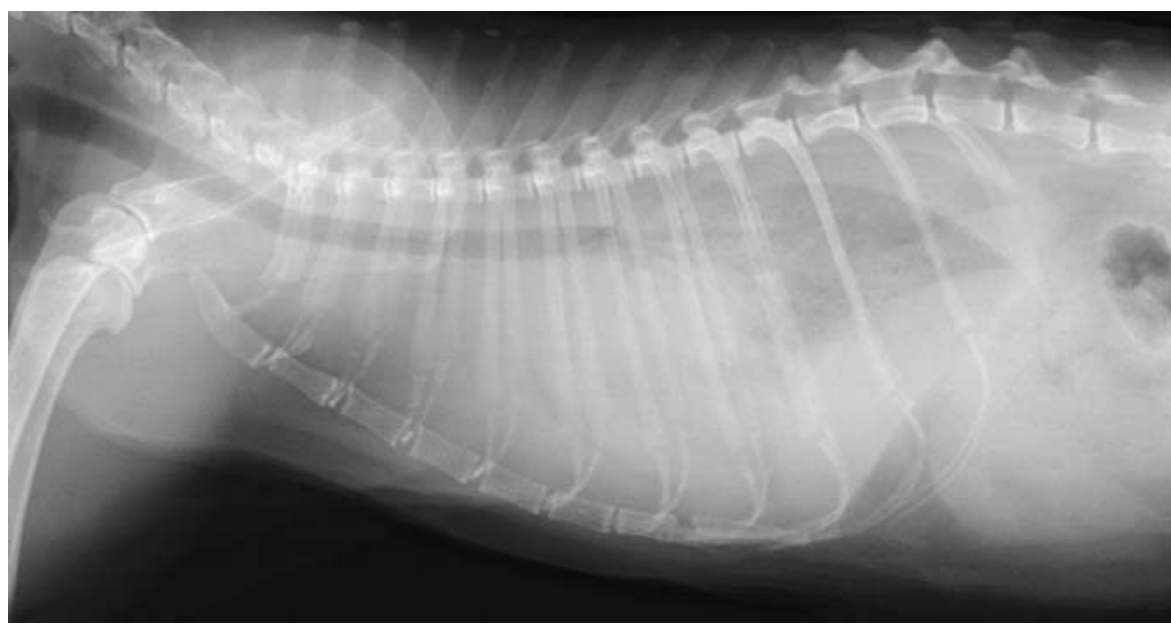

Abb. 2 Thoraxerguss infolge Linksherzversagens. Die Trachea ist aufgrund des Ergusses nach dorsal verschoben. ( $)$ M. Killich

urteilung des felinen Herzens in vielen Fällen nicht so einfach möglich wie beim Hund. Beispielsweise zeichnet sich das linke Atrium bei der Katze im latero-lateralen Röntgengang aufgrund seiner Lage im Thorax nicht so gut ab wie beim Hund. Die klassische Deviation des linken Hauptbronchus nach dorsal als Folge einer linksatrialen Vergrößerung ist bei der Katze nicht zu erwarten. Eine linksatriale Dilatation ist besser im ventrodorsalen oder dorsoventralen Strahlengang zu erkennen. Ein dilatiertes linkes Atrium spreizt dabei die beiden Hauptbronchien auseinander.

Eine weitere Schwierigkeit ist, dass Katzen im Gegensatz zum Hund, bei dem ein kardiales Lungenödem immer im perihilären oder caudodorsalen Lungenfeld beginnt, keine klassische Verteilung bei einem
Lungenödem zeigen ( $\bullet$ Abb. 1). Bei Patienten mit Pleuralerguss ist die Trachea häufig nach dorsal deviiert, was jedoch nicht auf eine kardiale Vergrößerung, sondern auf ein Nach-dorsal-Schwimmen der Lunge auf dem Erguss zurückzuführen ist ( $\boldsymbol{O}$ Abb. 2).

\section{Echokardiografie}

Die Echokardiografie ist nach wie vor der diagnostische Goldstandard. Fast in allen Fällen sind Septum, linke freie Wand oder beide linksseitigen Kammerwände hypertrophiert, nur selten ist auch die rechte freie Wand betroffen. Insgesamt präsentiert sich die Erkrankung äußerst heterogen. Das phänotypische Bild reicht von einer lokal begrenzten Hypertrophie bis hin zu einer
Echokardiokardiografische

Veränderungen bei einer HCM

- enddiastolische Wandstärken >6,0 mm

- Papillarmuskelhypertrophie

- endsystolische Kammerobliteration

- \pm linksatriale Dilatation

- \pm systolic anterior motion (SAM)

- diastolische Dysfunktion

generalisierten Hypertrophie von Septum und linker freier Wand ( $\mathbf{O}$ Abb. 3) [18].

Enddiastolisch gemessene linksventrikuläre Wandstärken von mehr als $6,0 \mathrm{~mm}$ sprechen für eine Hypertrophie, Patienten mit enddiastolischen Wandstärken zwischen 5,0 und $6,0 \mathrm{~mm}$ liegen im Graubereich (equivocal) und sollten im Zweifel erneut kontrolliert werden.

Nicht selten manifestiert sich die Erkrankung auch als Papillarmuskelhypertrophie. Diese kann ein erstes Anzeichen der Erkrankung sein oder später im Verlauf der Krankheit auftreten. Die Beurteilung der Papillarmuskeln ist jedoch nicht einfach, da bisher kein objektivierbares Messverfahren existiert, mit dessen Hilfe ihre Größe ausgemessen werden kann.

Echokardiografische Veränderungen, die für eine HCM sprechen, sind im $\bullet$ Kasten oben abgebildet.

Systolic anterior motion (SAM) beschreibt ein Phänomen, bei dem Teile des Mitralklappen-Apparates in den linksventrikulären Ausflusstrakt eingesogen werden und so zu einer dynamischen AusflusstraktObstruktion führen ( Abb.4). Die Kombi-
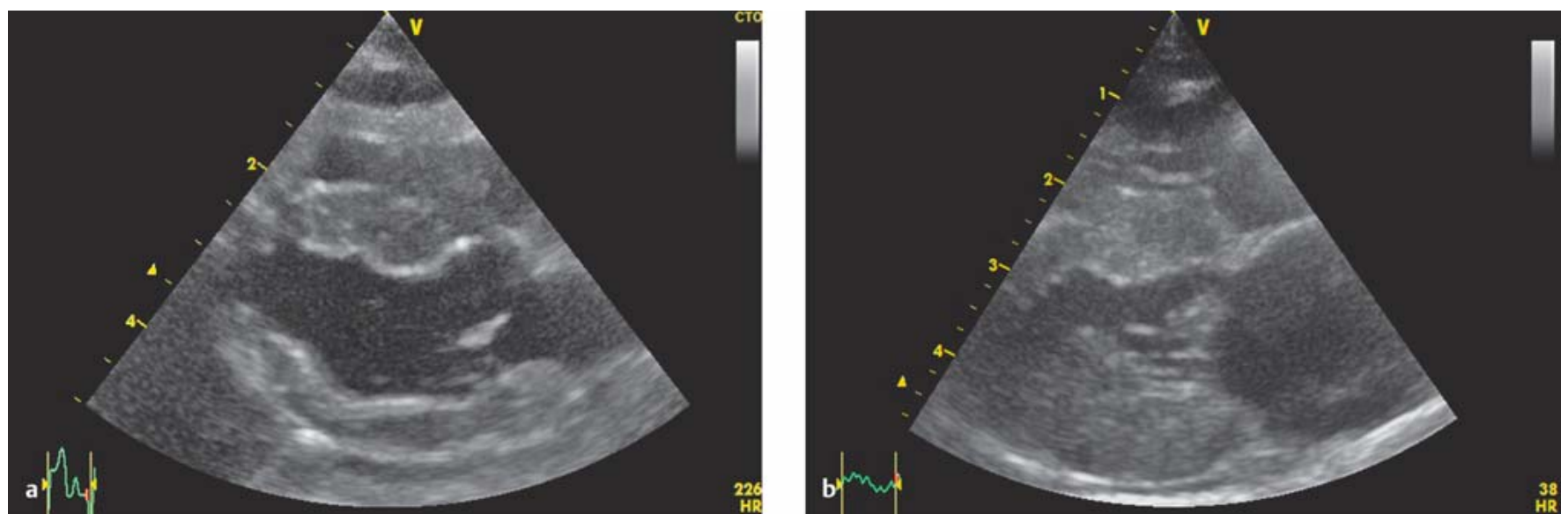

Abb. $\mathbf{3}$ a und b a fokale septale Hypertrophie, b generalisierte linksventrikuläre Hypertrophie. @ M. Killich 

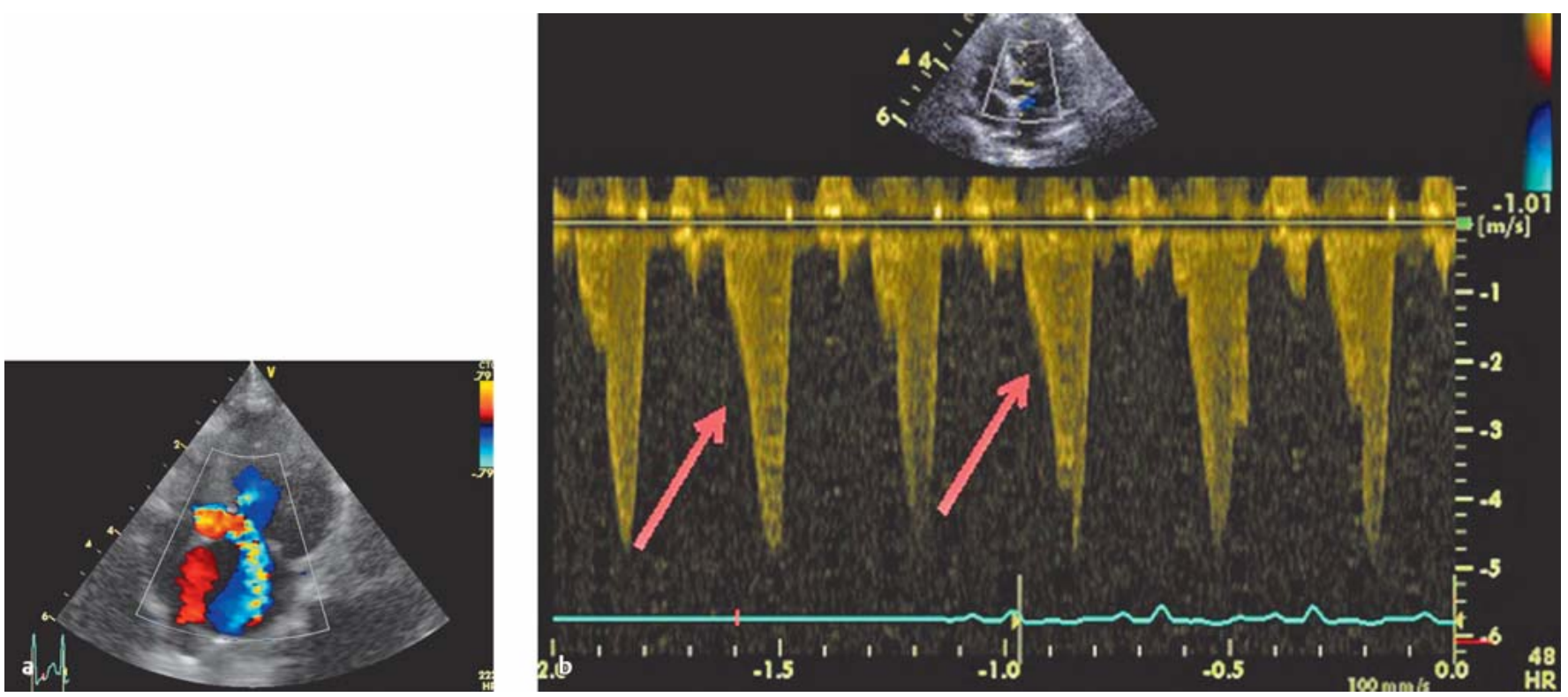

Abb. 4 a und b a SAM - Systolic anterior motion im Farbdoppler - dynamische Ausflusstrakt-Obstruktion und Mitralregurgitation; b charakteristisches, „dolch-förmiges“ Flussprofil einer dynamischen Ausflusstrakt-Obstruktion über der Aorta (CW-Doppler). @ M. Killich

nation von HCM und SAM wird oft auch als hypertroph obstruktive Kardiomyopathie (HOKM, HOCM) bezeichnet. Beim Menschen korreliert ein Druckgradient von mehr als $30 \mathrm{~mm} \mathrm{Hg}$ über dem linken Ausflusstrakt mit einer schlechten Prognose [15].

Die systolische Funktion ist bei den meisten Patienten erhalten, teils sind erkrankte Herzen sogar hyperkontraktil. Selten stellt sich im Endstadium der Erkrankung allerdings eine schwere systolische Dysfunktion ein, die Burn-out-Kardiomyopathie ( $\triangle$ Abb.5). Eine diastolische Dysfunktion ist dagegen eine frühzeitig auftretende Veränderung ( $\mathbf{O} \mathbf{A b b}$. $\mathbf{6})$. Leider hat die Echokardiografie hier ihre Grenzen, weshalb ihr Nachweis nicht immer einfach ist. Veränderungen im Mitraleinfluss-Profil, bei der isovolumischen Relaxation oder bei der Dezelerations-Zeit deuten auf eine gestörte diastolische Funktion hin. Gerade neueren Doppler-Techniken wie dem Gewebedoppler kann in der Früherkennung eine interessante Aufgabe zukommen $[4,9$, 11].

Die linksatriale Größe gilt als einfacher Indikator für Schweregrad und Chronizität der Erkrankung. Das bedeutet vereinfacht, je Größer der linke Vorhof, umso höher der Schweregrad und umso schlechter die Prognose. Eine Besonderheit bei Katzen ist, dass 


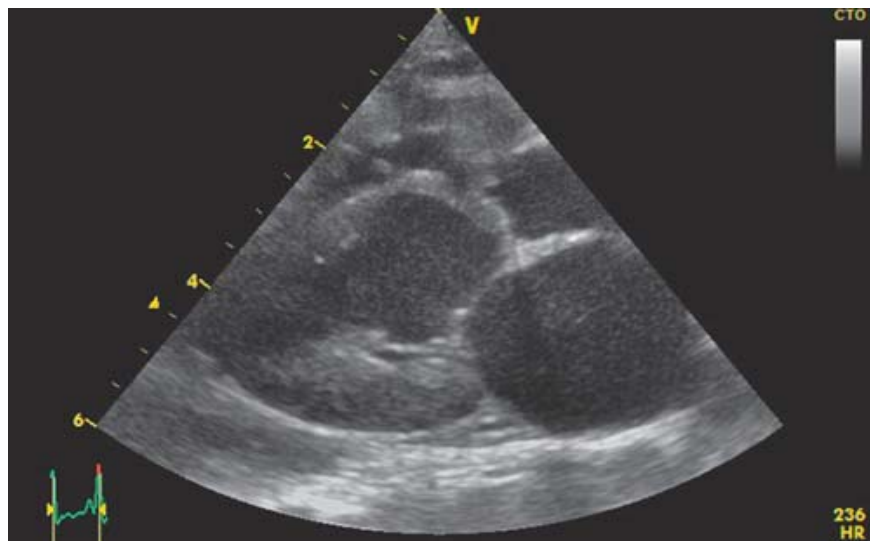

Abb. 5 Burn-outKardiomyopathie: das Septum ist ausgedünnt und der linke Ventrikel erscheint balloniert. (c) M. Killich

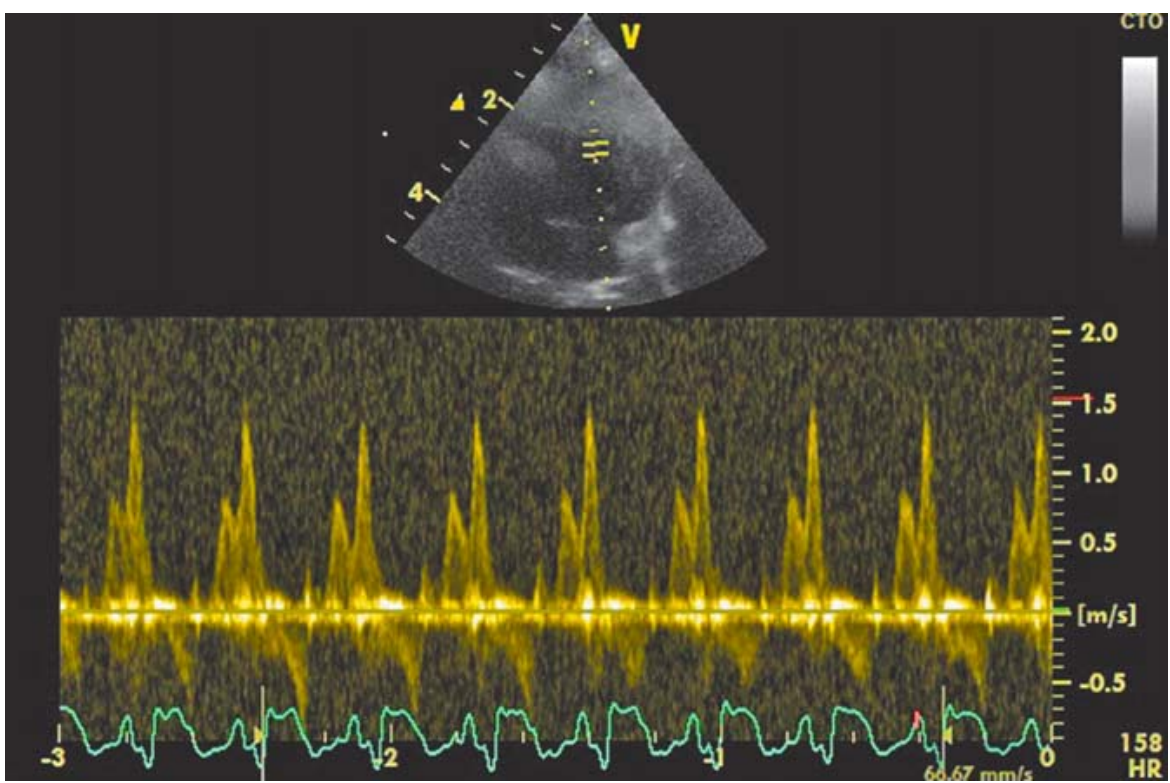

Abb. 6 Frühes Stadium der diastolischen Dysfunktion (Grad I, gestörte Relaxation). Die E-Welle ist hier größer als die A-Welle. (c) M. Killich

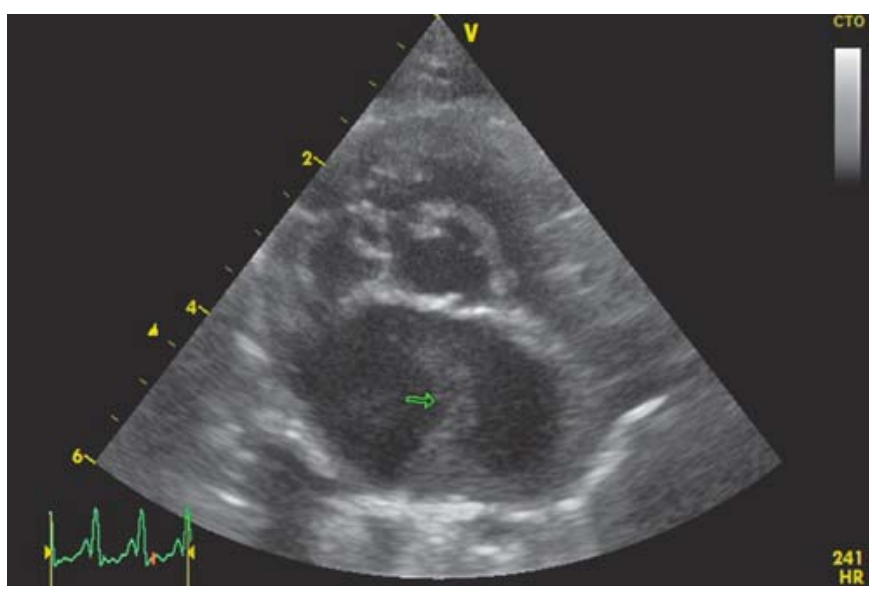

Abb. 7 Smoke im linken Atrium; gerinnendes Blut stellt sich als Schlieren-Bildung im linken Atrium dar (Pfeil). (C) M. Killich diese im Rahmen eines kongestiven Herzversagens häufig einen milden Perikarderguss aufweisen. Tatsächlich ist die häufigste Ursache für einen Perikarderguss bei Katzen ein kongestives Herzversagen [1].

Die HCM präsentiert sich echokardiografisch sehr heterogen, von fokalen Wandverdickungen bis hin zu einer globalen linksventrikulären Hypertrophie. Ein geeigneter Indikator für den Schweregrad ist die linksatriale Größe.

Die häufigste Ursache für einen Perikarderguss bei Katzen ist ein kongestives Herzversagen.

\section{Biomarker und Gentest}

Biomarker. Die Messung des Biomarkers NTProBNP erscheint für den Praxisalltag sinnvoll. So ist eine Unterscheidung von kardial bedingter Atemnot und nicht-kardial bedingter Atemnot möglich [3]. Auch lassen sich Katzen mit okkulter HCM anhand erhöhter Spiegel von herzgesunden Patienten unterscheiden [19].

Beispielweise kann der NT-pro-BNPSpiegel im Rahmen eines prä-anästhetischen Screening-Profils bestimmt werden. Ist der Marker erhöht, sollte vor einer anstehenden Operation eine kardiologische Abklärung mittels Herzultraschall erfolgen. Bei Werten innerhalb des Referenzbereichs liegt dagegen mit hoher Wahrscheinlichkeit keine Herzerkrankung vor. Ein Nachteil des Tests ist, dass milde Stadien der Erkrankung nicht erkannt werden. Ein Einsatz zum Screening bei Zuchttieren ist deshalb nicht anzuraten.

Gentest. Der Nutzen von Gentests zum Screening ist dagegen nach wie vor umstritten, da einerseits auch homozygot positive Katzen phänotypisch gesund bleiben können und andererseits Katzen mit HCM oft keine nachweisbare Genveränderung aufwiesen [20]. Eine Ursache dafür, dass homozygot positive Tiere keine HCM ausbilden müssen, ist, dass die phänotypische Ausprägung durch modifizierende Gene und Umweltfaktoren mitbeeinflusst zu sein scheint. 
NT-pro-BNP kann bedingt zum Screening nach Patienten mit HCM eingesetzt werden. Nachteilig ist, dass milde Erkrankungsstadien nicht erkannt werden. Der Nutzen von Gentests bleibt dagegen eingeschränkt.

\section{Therapie}

Die therapeutischen Grundpfeiler im chronischen kongestiven Herzversagen sind ACE-Hemmer und Furosemid [2]. Allerdings existiert bisher keine wissenschaftliche Studie, die einen positiven Effekt von ACEHemmern, Aldosteronantagonisten oder einem anderen Medikament auf den Krankheitsverlauf in früheren asymptomatischen Stadien belegt ( $\odot$ Tab. 1) [12,13].
Ausblick in die Humanmedizin. Auch beim Menschen fehlt dieser Nachweis gegenwärtig. Humanpatienten mit asymptomatischer HCM werden nicht behandelt [14]. Symptomatische Menschen mit obstruktiver HCM werden mit Beta-Blockern oder seltener einem Kalzium-Kanal-Blocker therapiert. Das Ziel ist eine Linderung der Symptome. Kann dieses nicht medikamentös erzielt werden, wird in der Humanmedizin eine Katheter-Alkohol-Ablation oder in manchen Fällen auch chirurgisch eine Myektomie durchgeführt [14]. Studien, die eine Verzögerung des Krankheitsverlaufs durch eine medikamentöse Intervention zeigen, gibt es auch in der Humanmedizin keine.
Tab. 1 Therapie der hypertrophen Kardiomyopathie (- keine Behandlung, + indiziert).

\begin{tabular}{|c|c|c|c|}
\hline \multirow[t]{2}{*}{ Stadium } & \multicolumn{3}{|c|}{ Therapie } \\
\hline & $\begin{array}{l}\text { ACE- } \\
\text { Hemmer }\end{array}$ & $\begin{array}{l}\text { Furose- } \\
\text { mid }\end{array}$ & $\begin{array}{l}\text { Atenolol/ } \\
\text { Diltiazem }\end{array}$ \\
\hline A & - & - & - \\
\hline B & - & - & $\begin{array}{l}\text { Tachykar- } \\
\text { die/SAM }\end{array}$ \\
\hline C & + & + & $\begin{array}{l}\text { Tachykar- } \\
\text { die/SAM* }\end{array}$ \\
\hline
\end{tabular}

Stadium A: erhöhtes Risiko, an einer HCM zu erkranken; Stadium B: erkrankt aber asymptomatisch;

Stadium C: kongestives Herzversagen;

* Atenolol im Herzversagen nur mit Vorsicht einsetzen 
Tachykardien oder obstruktive Kardiomyopathien werden bei Katzen ähnlich wie beim Menschen mit Atenolol oder Diltiazem behandelt. Vorsichtig sollte man allerdings beim kongestiven Herzversagen sein, da Atenolol in diesem Stadium die Überlebenszeit verkürzte [2].

Pimobendan $(0,125 \mathrm{mg} / \mathrm{kg}, 2 \times \mathrm{tgl}$.) findet bei der Behandlung der HCM bisher nur in seltenen Fällen Anwendung, beispielsweise in der Therapie der Burn-out-Kardiomyopathie. Eine kürzlich erschienene Studie belegt allerdings, dass Pimobendan auch von Katzen mit HCM und regionaler Hypokinesie gut vertragen wird [5].

Der Einsatz von Spironolakton beruht bisher nur auf empirischen Erfahrungen. Auch wenn eine Therapie mit dem u.a. antifibrotisch wirkenden Medikament logisch erscheint, konnte die Wirksamkeit des Aldosteron-Antagonisten bei an HCM-erkrankten Maine Coon nicht nachgewiesen werden [12].

Beim bisher oft frustrierenden Kampf gegen die gefürchtete Aortenthrombose steht mit dem Thrombozyten-AggregationsHemmer Clopidogrel ein neues, viel versprechendes Medikament zur Verfügung. In der kürzlich veröffentlichen FAT-CATStudie war Clopidogrel (18,5 mg/Katze $1 \times$ tgl.) gegenüber Aspirin überlegen [8]. Aspirin sollte bei Katzen aufgrund mangelnder Effektivität daher nicht mehr zur Thromboseprophylaxe eingesetzt werden. Wird Clopidogrel nicht vertragen, sollte eher auf Fragmin (100 IU/kg 2-3 × tgl.) zurückgegriffen werden. Ein Antikoagulans sollte bei allen Patienten mit deutlich vergrößertem linken Atrium, spätestens bei Auftreten von linksatrialem Smoke eingesetzt werden (@ Abb. 7)

Die Therapie der felinen HCM zielt auf eine Verringerung der Risikofaktoren (Thrombembolie, Leistungsintoleranz durch HOCM, plötzlicher Herztod durch Tachyarrhythmien) und nicht auf ein Aufhalten des Krankheitsprozesses ab.

\section{Literatur}

1 Davidson BJ, Paling AC, Lahmers SL, Nelson OL. Disease association and clinical assessment of feline pericardial effusion. J Am Anim Hosp Assoc 2008; 44: 5-9

2 Fox PR. Prospective, double-blinded, multicenter evaluation of chronic therapies for feline diastolic heart failure: interim analysis. In: American College of Veterinary Internal Medicine; 2003; Lakewood, CO; 2003

3 Fox PR, Oyama MA, Reynolds C, Rush JE, DeFrancesco TC, Keene BW et al. Utility of plasma $\mathrm{N}$-terminal pro-brain natriuretic peptide (NT-proBNP) to distinguish between congestive heart failure and non-cardiac causes of acute dyspnea in cats. J Vet Cardiol 2009; 11 (Suppl. 1): S51-S61

4 Gavaghan BJ, Kittleson MD, Fisher KJ, Kass PH, Gavaghan MA. Quantification of left ventricular diastolic wall motion by Doppler tissue imaging in healthy cats and cats with cardiomyopathy. Am J Vet Res 1999; 60: 1478-1486

5 Gordon SG, Saunders AB, Roland RM, Winter $R L$, Drourr L, Achen SE et al. Effect of oral administration of pimobendan in cats with heart failure. J Am Vet Med Assoc 2012; 241: 89-94

6 Granstrom S, Godiksen MT, Christiansen M, Pipper CB, Willesen JL, Koch J. Prevalence of hypertrophic cardiomyopathy in a cohort of British Shorthair cats in Denmark. J Vet Intern Med 2011; 25: 866-871

7 Gundler S, Tidholm A, Haggstrom J. Prevalence of myocardial hypertrophy in a population of asymptomatic Swedish Maine coon cats. Acta Vet Scand 2008; 50: 22

8 Hogan DF. Analysis of the Feline Arterial Thromboembolism: Clopidogrel vs. Aspirin Trial (Fat Cat). In: ACVIM Proccedings; 2013; Seatle; 2013

9 Koffas H, Dukes-McEwan J, Corcoran BM, Moran CM, French A, Sboros Vet al. Pulsed tissue Doppler imaging in normal cats and cats with hypertrophic cardiomyopathy. J Vet Intern Med 2006; 20: 65-77

10 Luis Fuentes $V$. Arterial thromboembolism: risks, realities and a rational first-line approach. J Feline Med Surg 2012; 14: 459-470

11 MacDonald KA, Kittleson MD, Kass PH, Meurs $K M$. Tissue Doppler imaging in Maine Coon cats with a mutation of myosin binding protein C with or without hypertrophy. J Vet Intern Med 2007; 21: 232-237

12 MacDonald KA, Kittleson MD, Kass PH, White $S D$. Effect of spironolactone on diastolic function and left ventricular mass in Maine Coon cats with familial hypertrophic cardiomyopathy. J Vet Intern Med 2008; 22: 335-341

13 MacDonald KA, Kittleson MD, Larson RF, Kass $P$, Klose T, Wisner ER. The effect of ramipril on left ventricular mass, myocardial fibrosis, diastolic function, and plasma neurohormones in Maine Coon cats with familial hy- pertrophic cardiomyopathy without heart failure. J Vet Intern Med 2006; 20: $1093-$ 1105

14 Maron BJ, McKenna WJ, Danielson GK, Kappenberger LJ, Kuhn HJ, Seidman CE, et al. American College of Cardiology/European Society of Cardiology Clinical Expert Consensus Document on Hypertrophic Cardiomyopathy. A report of the American College of Cardiology Foundation Task Force on Clinical Expert Consensus Documents and the European Society of Cardiology Committee for Practice Guidelines. Eur Heart J 2003; 24: 1965-1991

15 Maron MS, Olivotto I, Betocchi S, Casey SA, Lesser JR, Losi MA, et al. Effect of left ventricular outflow tract obstruction on clinical outcome in hypertrophic cardiomyopathy. N Engl J Med 2003; 348: 295-303

16 Paige CF, Abbott JA, Elvinger F, Pyle RL. Prevalence of cardiomyopathy in apparently healthy cats. J Am Vet Med Assoc 2009; 234: 1398-1403

17 Rush JE, Freeman LM, Fenollosa NK, Brown DJ. Population and survival characteristics of cats with hypertrophic cardiomyopathy: 260 cases (1990-1999). J Am Vet Med Assoc 2002; 220: 202-207

18 Trehiou-Sechi E, Tissier R, Gouni V, Misbach C, Petit AM, Balouka D et al. Comparative echocardiographic and clinical features of hypertrophic cardiomyopathy in 5 breeds of cats: a retrospective analysis of 344 cases (2001-2011). J Vet Intern Med 2012; 26: 532-541

19 Wess G, Daisenberger P, Mahling M, Hirschberger J, Hartmann $K$. Utility of measuring plasma N-terminal pro-brain natriuretic peptide in detecting hypertrophic cardiomyopathy and differentiating grades of severity in cats. Vet Clin Pathol 2011; 40: 237-244

20 Wess G, Schinner C, Weber K, Kuchenhoff $H$, Hartmann K. Association of A31P and A74 T polymorphisms in the myosin binding protein C3 gene and hypertrophic cardiomyopathy in Maine Coon and other breed cats. J Vet Intern Med 2011; 24: 527-532

\section{Online}

http://dx.doi.org/10.1055/s-0034-1382834

\section{Verfasser}

Dr. Markus Killich,

Diplomate ACVIM (Cardiology), Diplomate ECVIM-CA (Cardiology)

Olmützer Weg 1

85737 Ismaning

www.kardiokonsult.de 